\title{
Topiramate-Induced Acute Myopia, Diplopia, and Photosensitivity: A Case Report
}

\author{
Murat Kocamaz, (1) Onur Karadag
}

Department of Ophthalmology, Darica Farabi Training and Research Hospital, Kocaeli, Turkey

\begin{abstract}
Topiramate is primarily used as an antiepileptic drug. It has also demonstrated effectiveness in migraine prophylaxis, depression, trigeminal neuralgia, bipolar disorders, and idiopathic intracranial hypertension. The drug has a large number of potential ocular side effects, such as acute-onset angle closure glaucoma (ACG), acute myopia, nystagmus, diplopia, photosensitivity, suprachoroidal effusions, peri-orbital edema, and blepharospasm. Presently described is the case of a 24-year-old woman presenting with a sudden loss of vision in both eyes. Her medical history revealed that the patient had initiated topiramate use 12 days earlier. The uncorrected visual acuity was 20/400 in both eyes and the best corrected visual acuity was determined to be $20 / 25$ in the right eye with -5.50 spherical refractive correction and 20/20 in the left eye with -6.25 spherical refractive correction. On the second day of examination, diplopia developed. The patient's clinical condition was considered to be related to the drug and topiramate was discontinued immediately. The clinical findings of the patient subsequently improved rapidly without treatment. On the fifth day of examination, her autorefractometry measurements were $+0.25-0.25 \alpha 121^{\circ}$ in her right eye and +0.25 in her left eye and her uncorrected visual acuity was $20 / 20$ in both eyes with normal bilateral anterior chamber depth. She had no vision complaint or diplopia but she began to experience photosensitivity, which persisted for 4 months before regressing completely. When ophthalmologists encounter acute myopia and acute-onset ACG, especially in young patients, they should keep the use of topiramate in mind. Keywords: Acute myopia, diplopia, photosensitivity, topiramate.
\end{abstract}

\section{Introduction}

Topiramate is primarily used as an antiepileptic drug, both as monotherapy and as an adjunct therapy in the control of partial and primary generalized epilepsy in children and adults (I). Effectiveness has also been demonstrated in migraine prophylaxis, depression, trigeminal neuralgia, bipolar disorders, eating disorders, and idiopathic intracranial hypertension (2). Potential ocular side effects of acute-onset angle closure glaucoma (ACG), acute myopia, nystagmus, diplopia, suprachoroidal effusions, peri-orbital edema, scleritis, and blepharospasm have been reported in recent articles (3). This report describes a rare case with acute myopia, diplopia, and photosensitivity associated with topiramate use.

\section{Case Report}

The patient was a 24-year-old woman who presented with a sudden loss of vision in both eyes. According to her medical history, there were no vision disturbances and she had not even used glasses. Her uncorrected visual acuity was $20 / 400$ in both eyes and the best corrected visual acuity was 20/25 with -5.50 spherical refractive correction in her right eye and 20/20 with -6.25 spherical refractive correction in her left eye. Autorefractometry measurements after cycloplegia with $1 \%$ cyclopentolate hydrochloride eye drops were $-5.25-0.25 \alpha 120^{\circ}$ in her right eye and $-6.25-0.50 \propto 130^{\circ}$ in her left eye. Biomicroscopic examination revealed that the anterior chambers were very shallow bilaterally (Fig. I). A

Address for correspondence: Murat Kocamaz, MD. Darica Farabi Egitim ve Arastirma Hastanesi,

Oftalmoloji Bolumu, Kocaeli, Turkey

Phone: +90 5058168171 E-mail: muratko87@gmail.com

Submitted Date: October 10, 2018 Accepted Date: January 04, 2019 Available Online Date: February 20, 2019

${ }^{\odot}$ Copyright 2019 by Beyoglu Eye Training and Research Hospital - Available online at www.beyoglueye.com 


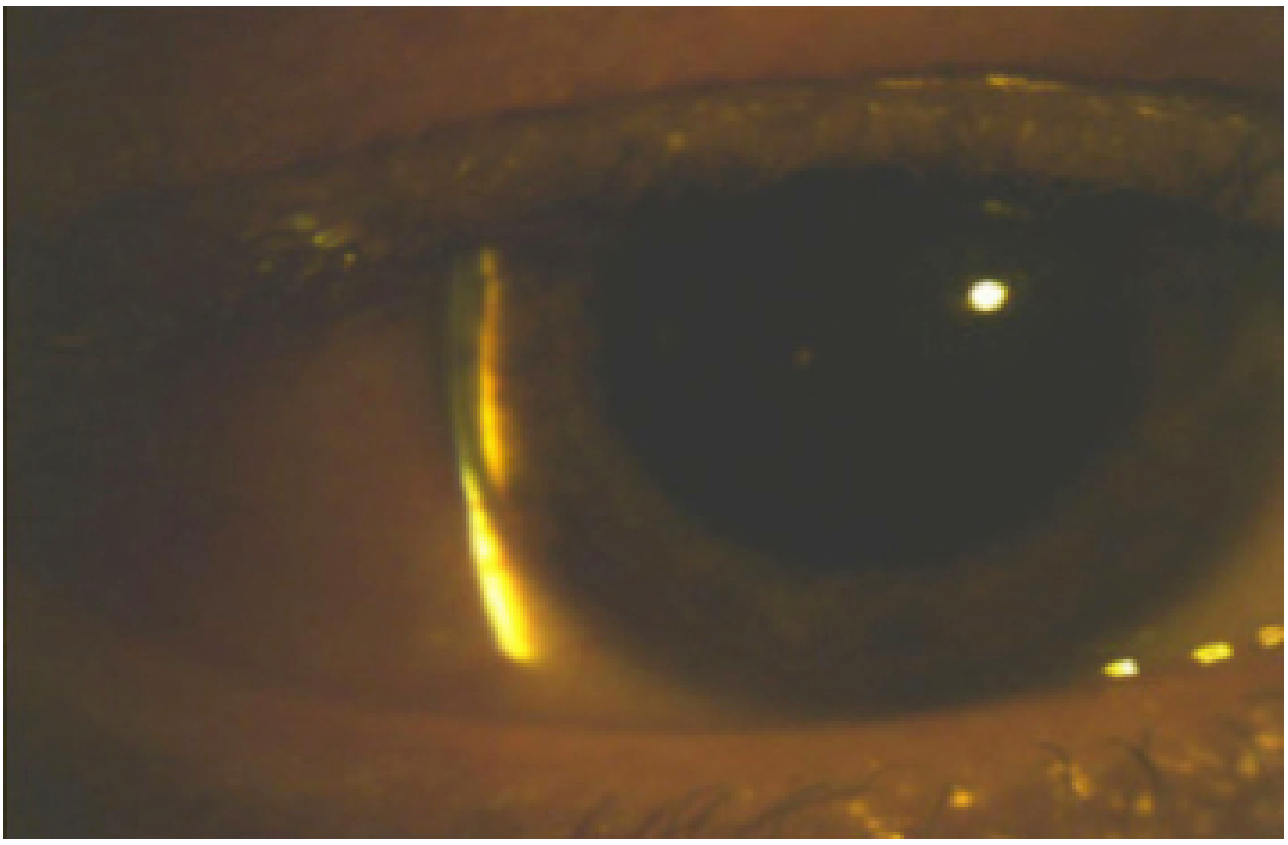

Figure I.Van Herick's technique revealed a grade 2 narrow angle in the patient's left eye.
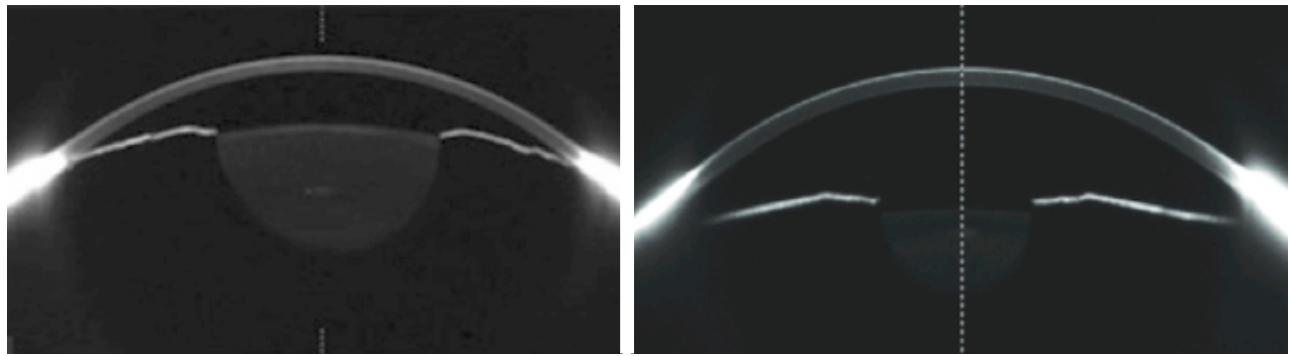

Figure 2. (a) Scheimpflug screening of the patient's right eye during the first examination revealed anterior chamber shallowness, lens thickening, and anterior transposition of the iris-lens diaphragm. The anterior chamber depth is $1.97 \mathrm{~mm}$ and the lens thickness is $4.74 \mathrm{~mm}$. (b) The patient's right eye as it appeared on the fifth day of discontinuation of the drug. The image shows a deepening of the anterior chamber and transposition of the iris-lens diaphragm to the usual position. The anterior chamber depth is $3.42 \mathrm{~mm}$ and the lens thickness is $4.27 \mathrm{~mm}$.

Scheimpflug image showed anterior chamber shallowness, lens thickening, and anterior transposition of the iris-lens diaphragm (Fig. 2a). Intraocular pressure assessed with a Goldmann applanation tonometer was $22 \mathrm{mmHg}$ in her right eye and $19 \mathrm{mmHg}$ in her left eye. A fundus examination was entirely normal; the cup-to-disc ratio in both eyes was 0.2 . Gonioscopy revealed grade 2 occludable angles bilaterally. The results of orbital magnetic resonance imaging were normal. The visual field test results were normal bilaterally, with no scotoma/visual field defect. When she was questioned about diseases and medications, it was discovered that she had epilepsy and that a neurologist had recently initiated the use of topiramate (Topamax, $25 \mathrm{mg}, 2 x$ daily). Twelve days later the patient began to experience blurred vision. With the thought that the patient's clinical status could be drugrelated, her neurologist was consulted and topiramate was replaced with another medication. The patient was examined daily and no additional medication was required. On the second day of examination, however, she complained of diplopia; her autorefractometry measurements were -4.00 $-0.25 \alpha 121^{\circ}$ in her right eye and $-3.50-0.50 \propto 130^{\circ}$ in her left eye. On the fifth day of examination, her autorefractometry measurements were $+0.25-0.25 \propto 121^{\circ}$ in her right eye and +0.25 in her left eye. Her uncorrected visual acuity was 20/20 in both eyes and the anterior chamber depth was normal (Fig. 2b). Intraocular pressure assessed with a Goldmann applanation tonometer was $17 \mathrm{mmHg}$ in the right eye and $15 \mathrm{mmHg}$ in the left eye. She had no vision complaint but she began to experience photosensitivity (pupil sizes were normal; the right and left pupil diameter was 2.I $\mathrm{mm}$ and $2.3 \mathrm{~mm}$, respectively, in photopic conditions). One month after her first visit, the ophthalmologic examination 
was completely normal, but the photosensitivity remained. The patient was advised to use polaroid glasses, and she was examined at monthly intervals. At the fourth month, the photosensitivity had receded completely.

\section{Discussion}

Topiramate is a sulfamate-substituted monosaccharide derived from $D$-fructose. It is used mainly as an antiepileptic drug for both monotherapy and adjunct therapy in the control of partial and primary generalized epilepsy in adults and children (I). Efficacy has also been reported in migraine prophylaxis, trigeminal neuralgia, bipolar disorders, depression, and eating disorders (2). Recently, it has been used in the treatment of idiopathic intracranial hypertension (4). It works via inactivation of sodium and/or calcium channels, hyperpolarization of $\mathrm{K}+$ currents, inhibition of kainate receptor-mediated conductance, and activation of gamma-aminobutyric acid postsynaptic receptors (5). In addition, it also has weak anti-carbonic anhydrase activity. The mean plasma elimination half-life of the drug is about 21 hours. The half-life of the drug is closely related to the return of ocular side effects after discontinuation of the drug (5). Recent articles have described ocular side effects of topiramate, which include acute angle closure glaucoma (AACG), acute myopia, diplopia, blepharospasm, suprachoroidal effusions, peri-orbital edema, scleritis, oculogyric crisis, periocular pain, and nystagmus (3). The exact mechanism by which topiramate initially triggers myopia is not completely understood. To date, a number of mechanisms have been suggested as possible triggers. One proposed mechanism is that fluid movements result in uveal and ciliary effusion because of the ability of topiramate to block sodium channels and change the membrane potential (6). Sen et al. (7) suggested that the entry of topiramate into the lens alters the osmotic status of the lens, causes the lens to swell and, consequently, results in ACG and myopia. In 2002, Ikeda (8) described side effects of topiramate therapy ranging from transient myopia to severe bilateral ACG. These complications attributed to ciliochoroidal effusion and swelling of the ciliary body can result in anterior rotation of the ciliary processes, narrowing of the ciliary sulcus, and forward displacement of the iris and lens. This has been demonstrated using standard or high frequency ultrasound in patients using topiramate (3). Another theory is that ciliochoroidal effusion syndrome may be due to an idiosyncratic reaction. This complication occurs at a low frequency, and some studies have reported no correlation between topiramate dosage and the level of intraocular pressure or myopia (3). Some studies have suggested that the onset of AACG with topiramate use is not dose-related and that AACG occurs with doses ranging between $50 \mathrm{mg}$ to more than $100 \mathrm{mg}$ topiramate (3). In our case, the patient's complaints began on the $12^{\text {th }}$ day of $50 \mathrm{mg} /$ day topiramate use.
Furthermore, pre-existing hypermetropia is not a prerequisite for development of AACG secondary to topiramate therapy (9). The incidence or prevalence of AACG in the population of patients treated with topiramate is not known. The condition has predominantly been reported in women $(89 \%)(10)$. Some of these patients may be using selective serotonin reuptake inhibitors, which may aggravate the glaucoma by adding an element of pupillary block (II). Topiramate-induced AACG usually occurs within 2 weeks of the initiation of treatment (II). A number of patients taking topiramate have presented with characteristic findings of an acute attack of ACG, including blurred vision, headaches, nausea, and vomiting.

The acute myopia associated with using topiramate has been reported at between 2 and 8.75 diopters and causing sudden bilateral blurring of vision (12). Our case had -5.50 diopter myopia in her right eye and -6.25 diopter myopia in her left eye. The severity of ciliary body edema, ciliochoroidal detachment, and forward movement of the iris lens diaphragm causes myopia. Myopia on its own resolves following discontinuation of the drug, but occasionally myopia may persist after resolution of AACG. Drug-induced myopia has also been associated with sulfa drugs, such as acetazolamide, sulfamethoxazole/trimethoprim, indapamide, promethazine, spironolactone, isosorbide dinitrate, bromocriptine, as well as other drugs, including tetracycline, corticosteroids, hydrochlorothiazide, penicillamine, quinine, metronidazole, isotretinoin, and aspirin (13).

Diplopia and nystagmus have been reported in $14 \%$ to $15 \%$ of patients using high doses of topiramate (3). Although the dose of the drug was low ( $25 \mathrm{mg} 2 x$ daily) in our case, diplopia developed. Scleritis has been reported in 4 cases, oculogyric crisis in 2 cases, and single cases of blepharospasm, myokymia, periocular edema, paresthesia, and periocular pain have been described (3). A recent report demonstrated visual field defects with the usage of topiramate without the presence of elevated intraocular pressure (14).

A photosensitivity reaction following topiramate use has been more frequently observed in females $(80 \%)$ and the condition is more common in patients between 30 and 59 years old (15). Photosensitivity is usually detected among people who have been taking the drug for I to 6 months (I5). In the literature, the incidence of photosensitivity in patients using topiramate was found to be $0.12 \%$ (15). The mechanism of photosensitivity seen in patients using topiramate is not known (15). The photosensitivity in our patient began on the $10^{\text {th }}$ day of usage and ended in the fourth month of discontinuation of the drug.

In the case of topiramate-associated AACG and acute myopia development, the drug should be discontinued immediately and an alternative drug should be prescribed by the primary physician. The initial treatment should include cyclo- 
plegic agents to posteriorly displace the iris-lens diaphragm. Furthermore, topical and systemic ocular hypotensives and topical steroids can be used. Acetazolamide, a sulfonamide derivative drug, is not recommended for fear of further ciliary body edema. Also, topical miotics are contraindicated as a treatment option because they can cause further displacement of the iris-lens diagram, and they can lead to a relative pupillary block and worsen the ACG.

Peripheral iridotomy is not recommended in drug-induced secondary angle closure because the mechanism of this condition is not pupillary block; therefore, it has no therapeutic value in these cases. Laser peripheral iridotomy has been used in $23 \%$ of reported cases, but has not been sufficiently effective in relieving the secondary angle closure and should therefore be reserved for cases where the other treatments fail $(3,9)$. Topical steroids may be beneficial to reduce choroidal effusion by stabilizing cell membranes. Rapid resolution of an attack has been reported with the use of intravenous methylprednisolone and mannitol (16).

Parents of children and other patients who are to start topiramate treatment should be warned of the possible ocular side effects. Symptoms such as myopia and angle closure should be resolved with topical and oral intraocular pressure lowering medications, topical cyclopentolate, and discontinuation of topiramate. Physicians should inform patients to promptly report any symptoms of eye pain or blurred vision after commencing topiramate therapy, since if the symptoms remain unrecognized as a drug-related event, permanent ocular damage can occur. When ophthalmologists encounter acute myopia and acute angle closure glaucoma, especially in young female patients, they should closely examine the medical history of the patient and keep the use of topiramate in mind.

\section{Disclosures}

Informed consent: Written informed consent was obtained from the patient for the publication of the case report and the accompanying images.

Peer-review: Externally peer-reviewed.

Conflict of Interest: None declared.

Authorship Contributions: Involved in design and conduct of the study (MK); preparation and review of the study (MK, OK); data collection (MK, OK).

\section{References}

I. Rosenfeld WE, Sachdeo RC, Faught RE, Privitera M. Longterm experience with topiramate as adjunctive therapy and as monotherapy in patients with partial onset seizures: retrospective survey of open-label treatment. Epilepsia 1997;38 Suppl I:S34-6. [CrossRef]

2. Brandes JL, Saper JR, Diamond M, Couch JR, Lewis DW, Schmitt J, et al. Topiramate for migraine prevention: a randomized controlled trial. JAMA 2004;291:965-73. [CrossRef]

3. Fraunfelder FW, Fraunfelder FT, Keates EU. Topiramate-associated acute, bilateral, secondary angle-closure glaucoma. Ophthalmology 2004; I I I:109-I I. [CrossRef]

4. Randhawa S, Van Stavern GP. Idiopathic intracranial hypertension (pseudotumor cerebri). Curr Opin Ophthalmol 2008; 19:445-53. [CrossRef]

5. Langtry HD, Gillis JC, Davis R. Topiramate. A review of its pharmacodynamic and pharmacokinetic properties and clinical efficacy in the management of epilepsy. Drugs 1997;54:752-73.

6. Topamax side effects - from FDA reports. Available at: https:// www.ehealthme.com/drug/topamax/side-effects/. Accessed Feb 20, 2019.

7. Sen HA, O'Halloran HS, Lee WB. Case reports and small case series: topiramate-induced acute myopia and retinal striae. Arch Ophthalmol 2001; I 19:775-7.

8. Ikeda N, Ikeda T, Nagata M, Mimura O. Ciliochoroidal effusion syndrome induced by sulfa derivatives. Arch Ophthalmol 2002; 120: 1775.

9. Banta JT, Hoffman K, Budenz DL, Ceballos E, Greenfield DS. Presumed topiramate-induced bilateral acute angle-closure glaucoma. Am J Ophthalmol 200 I; 132: I I 2-4. [CrossRef]

10. Thambi L, Kapcala LP, Chambers W, Nourjah P, Beitz J, Chen M, et al. Topiramate-associated secondary angle-closure glaucoma: a case series. Arch Ophthalmol 2002; 1 20: I 108. [CrossRef]

I I. Craig JE, Ong TJ, Louis DL, Wells JM. Mechanism of topiramate-induced acute-onset myopia and angle closure glaucoma. Am J Ophthalmol 2004; 137:193-5. [CrossRef]

12. Myopia and glaucoma with topiramate. Prescrire Int 2003; 12:6I.

13. Bovino JA, Marcus DF. The mechanism of transient myopia induced by sulfonamide therapy. Am J Ophthalmol 1982;94:99102. [CrossRef]

14. Guier CP. Elevated intraocular pressure and myopic shift linked to topiramate use. Optom Vis Sci 2007;84:1070-3. [CrossRef]

15. Who have Photosensitivity reaction with Topamax - from FDA reports. Available at: https://www.ehealthme.com/ds/topamax/ photosensitivity-reaction/. Accessed Feb 20, 2019.

16. Rhee DJ, Ramos-Esteban JC, Nipper KS. Rapid resolution of topiramate-induced angle-closure glaucoma with methylprednisolone and mannitol. Am J Ophthalmol 2006; |4 I: | |33-4. 